\title{
Motion parallel to line orientation: Disambiguation of motion percepts
}

\section{Gregory Francis, Hyungjun Kim}

Department of Psychological Sciences, Purdue University, 1364 Psychological Sciences Building, West Lafayette, IN 47907-1364, USA; e-mail: gfrancis@psych.purdue.edu

Received 12 November 1998, in revised form 19 June 1999

\begin{abstract}
Four experiments demonstrate that lines indicating path of movement can generate rotational percepts in a multistable motion display that usually produces only horizontal or vertical motion percepts. The properties of the path-of-movement lines are predicted by a neuralnetwork theory of visual perception. Experimental results validate the theory's predictions by demonstrating that movement of the display elements seems to follow an increasing luminance gradient in lines but not bars, and that illusory contours have similar effects. Experimental results also demonstrate that, in a choice between movement along lines drawn parallel or orthogonal to possible motion paths, observers more often see movement along the lines parallel to the motion path. These results suggest modifications to current computational and neurophysiological theories of motion perception.
\end{abstract}

\section{Introduction}

Despite its cartoonish style, the drawing in figure 1a implies that the person is moving to the right. While familiarity with running postures and other cues undoubtedly influence people's judgments of implied motion in this drawing, the use of lines to the left of the figure is of particular note. These lines seem to indicate the path of motion traveled by the individual, and can be used in situations where the other cues are not applicable, as in figure $1 \mathrm{~b}$, where the lines indicate leftward movement of a circle. These motion lines are commonly used in cartoons to indicate motion direction and velocity (Carello et al 1986). The use of this motion indicator is interesting because, unlike other cues to motion in figure 1a, the motion lines seem to have no correlation with real moving objects. When a person runs, lines are not drawn behind him or her.

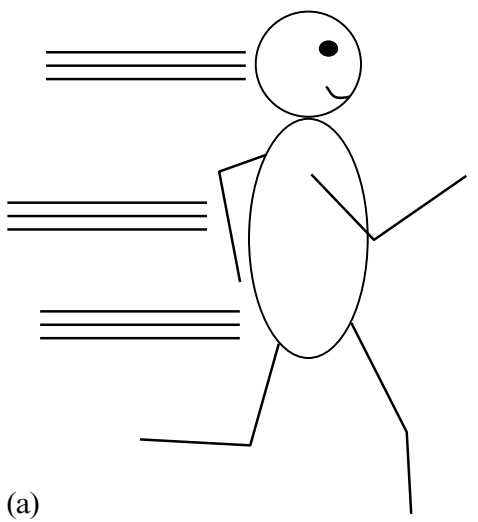

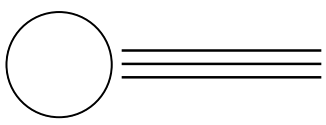

(b)

Kim and Francis (1998) accounted for the use of motion lines in drawn images with a neural-network theory of visual perception. In the neural-network theory, a moving oriented stimulus leaves a trail of responses among cells tuned to the orthogonal orientation. This trail provides information about movement direction and relative speed. It was hypothesized that drawn motion lines in images like figure 1 tap into 
some part of the visual system that interprets the oriented trail as a cue to motion, and thereby give an impression of motion parallel to the line orientation.

Kim and Francis (1998) also showed that motion lines could bias motion percepts in an otherwise ambiguous motion display. We now elaborate that type of experiment to test more precise predictions of the model. The next section summarizes the neural-network theory and the relationship of the network's rebound trail to motion lines. Subsequent sections then describe four experiments that test the theory.

\subsection{Rebound trails}

Figure 2 schematizes the parts of the neural-network theory that are most important for the current analysis. This circuit is a part of a larger network of circuits that are involved in preattentive processing of image contours called the boundary contour system (BCS) (Grossberg and Mingolla 1985; Grossberg 1994, 1997). The circuit in figure 2 includes separate pathways sensitive to the same position in visual space but perpendicular spatial orientations, which compete via reciprocal inhibition from lower levels to higher levels. Feeding this competition are inputs gated by habituative transmitters. Along with signals from external stimuli, each input pathway receives a tonic source of activity, and all output signals are rectified. This combination of rectification, cross-channel competition, habituative transmitter gates, and tonic input creates a gated dipole circuit (Grossberg 1972). At the offset of stimulation, a gated dipole circuit generates a transient rebound of activity in the previously nonstimulated pathway.

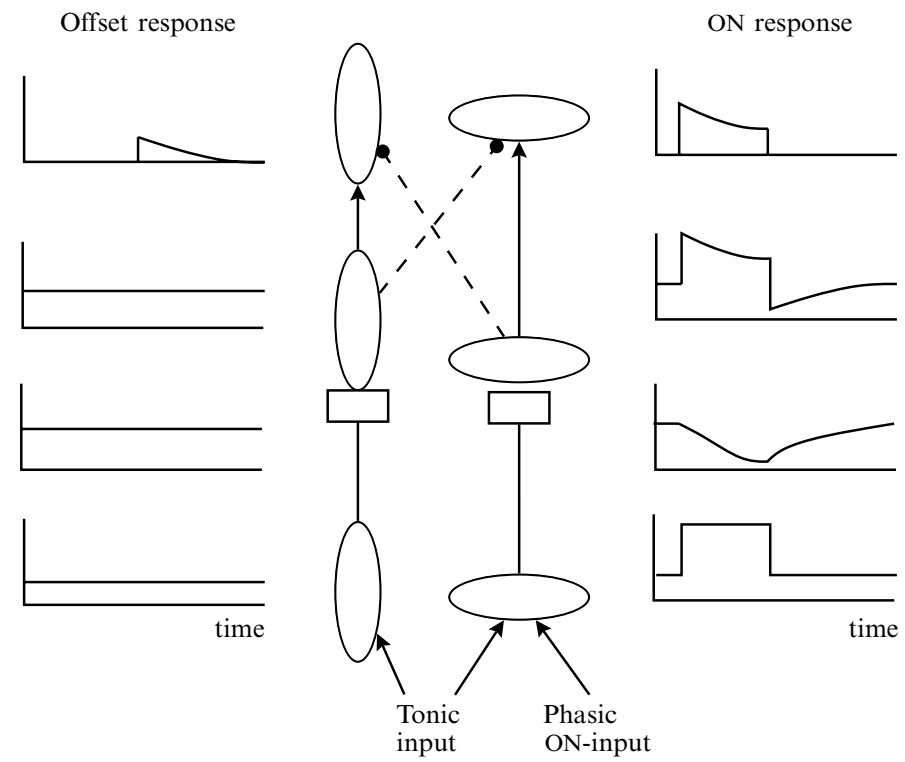

Figure 2. At stimulus offset, a gated dipole circuit produces a transient rebound of activity in the nonstimulated pathway. When the pathways code orthogonal orientations, offset of a horizontal input leads to a rebound of vertical activity. Dashed lines with circle terminators indicate inhibition, solid arrows indicate excitation, boxes indicate transmitter gates. The plot next to each cell or gate schematizes the signal strength over time as a horizontal input is applied and removed. Offset of the horizontal input leads to a rebound of activity in the vertical pathway.

The time plot next to each cell or gate describes the dynamics of this circuit. The sharp increase and then decrease of the time plot at the lower right of figure 2 indicates that an external input stimulates the horizontal pathway. This input produces both a response in the horizontal channel, and habituation in the horizontal channel. Thus, when the horizontal input turns off, the vertical channel wins the competition against the habituated horizontal channel to generate a rebound of activity. As the 
horizontal transmitter gate recovers from its habituated state, the rebound signal in the vertical channel weakens and finally disappears.

The oriented gated dipole rebounds are not an ad hoc addition to the BCS, but play an integral part in the control of excitatory feedback by inhibiting persisting responses that could otherwise lead to substantial smearing of changing stimuli. Details of the dynamic properties of the BCS and its relationship to psychophysical properties of visual persistence, temporal integration, apparent motion, oriented afterimages, and metacontrast masking can be found elsewhere (Francis et al 1994; Francis and Grossberg 1996a, 1996b; Francis 1996a, 1996b, 1997, 1999). A large literature on dynamic vision is consistent with the hypothesized role of the circuit in figure 2.

A recent neurophysiological study of macaque monkeys is also consistent with the behavior of gated dipole circuits tuned for orientation. Ringach et al (1997) explored dynamic changes in the orientation-tuning curves of cells. They measured cell action potentials during rapid presentation of a series of sine-wave gratings with different orientations. They then correlated action potentials with the grating orientations at successive times before each action potential. They found that action potentials correlated with one orientation after a short delay were also correlated with the orthogonal orientation after a longer delay. This finding is consistent with the properties of gated dipoles. The top vertically oriented cell in figure 2 is sensitive to the presentation of a vertical edge, but it also responds to the offset of a horizontal edge. The delay between the horizontal-edge offset and the vertical-cell response corresponds to the time needed for the rebound signal to develop.

After further analysis of the BCS model, Kim and Francis (1998) demonstrated that the rebounds created a cue that could be used for motion detection. Consider a vertical bar moving from left to right in the visual field. In the model, such a bar should produce gated dipole rebounds among horizontally tuned cells along its movement path, as schematized in figure 3. Such a trail of orientational rebounds contains information about the movement of the bar. For example, the relative strength of responses within a trail provides information about the direction of motion. The strongest responses will be closest to the stimulus, with weaker responses near where the bar started moving (figure 3c). Likewise, the speed of a moving bar is partly coded by the length of its rebound trail, with faster stimuli leaving longer trails. These properties were simulated by Kim and Francis (1998), and they proposed that the visual system is sensitive to the properties of rebound trails as indicators of motion direction and speed. In particular, responses of horizontally tuned cells along a horizontal line should be a cue that something has moved horizontally. The gradient of response

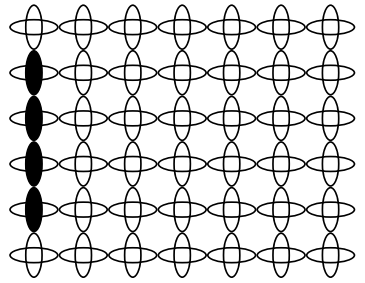

(a)

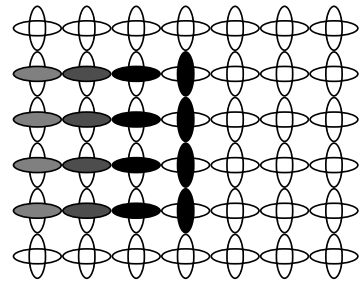

(b)

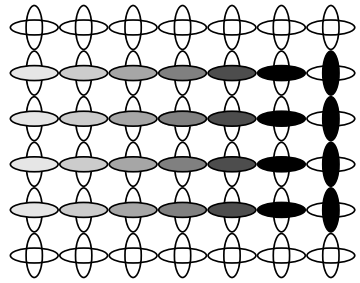

(c)

Figure 3. Hypothesized behavior of orientationally tuned cortical cells in response to a moving vertical bar. Darker shades of gray indicate stronger responses. (a) At the start of the movement, the bar excites a column of vertically tuned cells. (b) As the bar moves, the gated dipole competition between orientations generates a trail of activities among horizontally tuned cells. (c) As the bar moves further, the beginning parts of the trail grow weaker, thus creating a gradient of horizontal activities. We hypothesize that the visual system is tuned to the properties of this trail to identify stimulus motion direction and speed. 
strength along the line indicates the direction of the movement, and the length of the line gives a relative indication of the movement speed.

It should be noted that the trail of rebound signals does not correspond to a percept of blur as might be produced by very fast moving stimuli. The BCS interacts with a complementary system called the feature contour system (FCS) that codes percepts of brightness and color. The interactions between these system are complex, so that the offset of stimulus-generated signals in the BCS also corresponds to offset of responses in the FCS, but the appearance of orientational rebound signals does not produce FCS responses. Thus, while there is a 'smear' of rebound signals as a stimulus moves, this does not, in the model, indicate a percept of smeared images. Recent discussions of the interactions between the BCS and FCS can be found in Francis and Grossberg (1996b) and Grossberg (1997).

That the motion lines in figure 1 imply motion directions parallel to line orientation is consistent with the hypothesized role of oriented rebound signals in motion detection. In the next section, the hypothesis that a gradient of oriented responses will control perceived motion direction is tested in an otherwise ambiguous motion display.

\section{Experiment 1: Luminance gradient}

\subsection{Method}

All stimuli were presented on a Silicon Graphics Indy computer. Figure 4 schematizes, in reverse contrast, the type of stimuli used in all the experiments. Figure 4a schematizes the stimulus for one experimental condition. Frame 1 (far left) included two dots on opposite corners of an imaginary square. Frame 3 included two dots on opposite corners of the imaginary square, ie the corners not used in frame 1 . The dots were circles with a radius of $2.08 \mathrm{deg}$. The center-to-center distance of a dot from frame 1 to frame 3 was $5.56 \mathrm{deg}$. The dots had a luminance of $52 \mathrm{~cd} \mathrm{~m}^{-2}$ and the background was $0.06 \mathrm{~cd} \mathrm{~m}^{-2}$. Each frame was presented for approximately $150 \mathrm{~ms}$. The frames were presented in a loop so that frame 1 appeared right after frame 4. In a control condition (with frames 2 and 4 blank except for the fixation point) the percept was typically of dots moving back and forth, either horizontally or vertically. Though theoretically plausible, rotational motion has rarely been reported by observers in these types of displays (Ramachandran and Anstis 1985).

The theory predicts that adding lines with a luminance gradient should influence the motion percept by acting as a type of prime or bias for the movement of a dot. The theory predicts that adding lines to frames 2 and 4 as in figure $4 \mathrm{~b}$ will bias observers to report percepts of clockwise rotation. These lines include an intensity gradient that is from low to high luminance in a clockwise direction, within each line. The responses of orientationally tuned cells along these lines should increase with luminance, and the model predicts that a gradient of oriented responses is a cue to motion in the direction of the gradient increase.

For the lines experimental stimuli schematized in figure $4 b$, each set of lines consisted of nine lines with a thickness of $4.6 \mathrm{~min}$ of arc and a length of $3.4 \mathrm{deg}$. The low-luminance end of a line was $0.06 \mathrm{~cd} \mathrm{~m}^{-2}$ while the high-luminance end was $52 \mathrm{~cd} \mathrm{~m}^{-2}$. The intermediate luminances were linear HSB values between the two extremes. Figure $4 \mathrm{~b}$ schematizes a clockwise experimental stimulus. A counterclockwise lines stimulus included lines with the luminance gradient running in a counterclockwise direction.

A second experimental condition was designed to test whether a bar with the same luminance gradient would have any effect on perceived motion. Figure 4 a schematizes this type of stimulus. Differences between the two experimental conditions will indicate whether the intensity gradient alone or the lines with the gradient are most important in controlling the percept. 
(a)
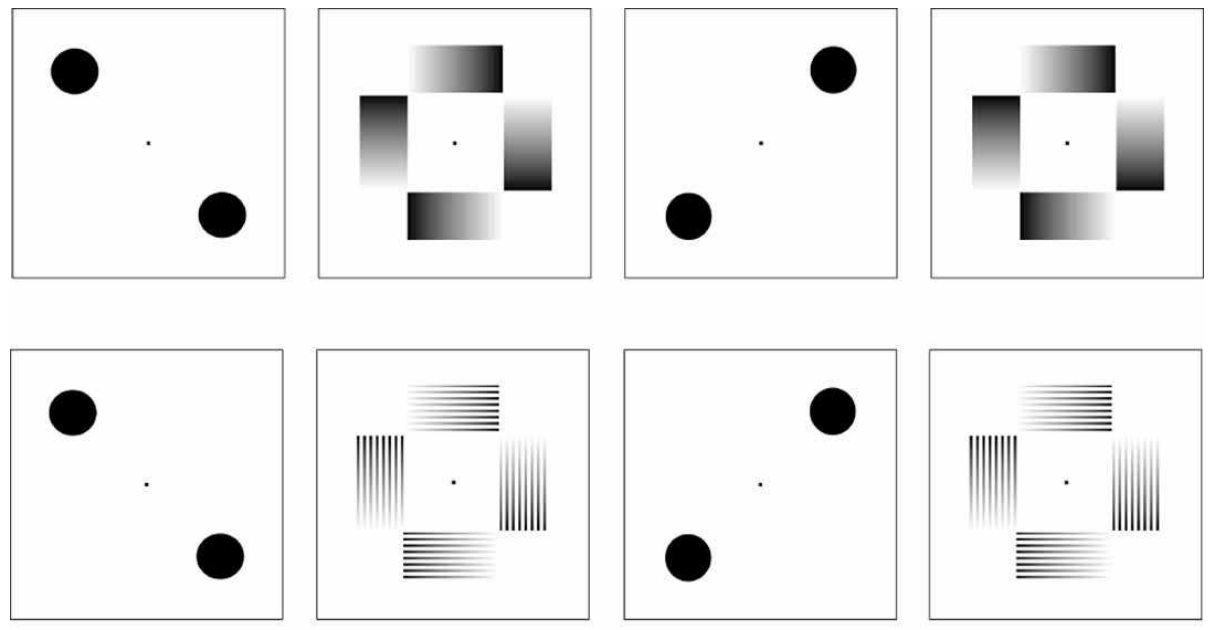

(b)
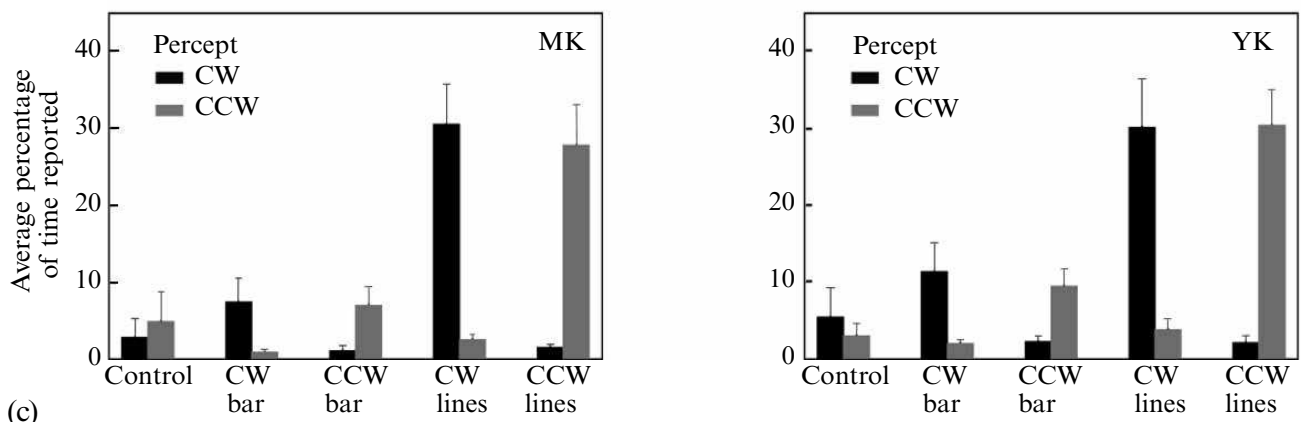

Figure 4. Experiment 1. All stimuli in (a) and (b) are the reverse contrast of what was on the screen. A control condition consisted of only a pair of dots presented in alternation, with the intervening frames containing only a fixation point. (a) The clockwise (CW) bar stimulus included bars with a clockwise gradient of intensities within each bar. (b) The CW lines stimulus included lines with the same gradient. (c) The percentage of time observers $\mathrm{MK}$ and $\mathrm{YK}$ reported seeing motions as indicated for the different stimuli. The control stimulus rarely produced rotational-motion percepts. Observers saw $\mathrm{CW}$ rotation for the clockwise lines stimuli and counterclockwise (CCW) rotation for the CCW lines stimuli, as predicted. The bar stimuli produced a small, but statistically insignificant, effect. Error bars indicate one standard error of the mean.

Two observers, naive to the purpose of the study, each participated in one testing session.

\subsection{Procedure}

In each trial, an observer viewed a repeating sequence of images from the control, lines or bar clockwise, or lines or bar counterclockwise conditions for $60 \mathrm{~s}$. The observer noted, by keypresses, whenever the percept changed to clockwise, counterclockwise, or some other type of motion. The computer kept track of the time that an observer saw the different types of motion. An observer first went through five practice trials of each condition. Data were then gathered from twenty trials for each of the conditions, which were randomly mixed as the observer went through the testing session.

\subsection{Results and discussion}

Figure $4 \mathrm{c}$ shows the average percentage of time across trials that observers reported rotational-motion percepts for the different conditions. In this figure, and all others, the error bars indicate standard error of the mean. For the control condition, both observers rarely reported rotational motion. The lines conditions led to more reports 
of rotational motion in a direction consistent with the model prediction. The clockwise increase in luminance gradient (CW lines) led to percepts of clockwise motion nearly $30 \%$ of the time. When the gradient was flipped to counterclockwise (CCW lines) the percept also flipped to counterclockwise motion nearly $30 \%$ of the time.

However, it is not the luminance gradient alone that is critical for producing the percept of rotational motion. For the bar conditions, much less rotational motion was observed. While there is a tendency for observers to report more rotational motion than in the control condition, the differences are not statistically significant when tested with the Tukey method $(p<0.05)$. The difference between the bar and lines conditions also indicates that it is not necessary for the spatial frequency of the influenced moving stimulus and the motion lines to match, as the bars and dots have more frequencies in common than the lines and dots. This is consistent with the model hypothesis that the neural representation of a stimulus involves responses from multiple cells that are sensitive to local parts of the stimulus contour (Grossberg and Mingolla 1985), as indicated in figure 3 where multiple cells code a vertical bar. Such an hypothesis does not preclude multiple scales among oriented filters, which are included in a significant extension of the BCS to create a larger theory of visual perception (Grossberg 1994, 1997).

The results of experiment 1 are quite remarkable with regard to current views of motion perception, and particularly the relationship between motion direction and spatial orientation. If the introduction of the stimuli in the intervening frames contributed to motion-energy or other Fourier-based methods (Adelson and Bergen 1985; Watson and Ahumada 1985; van Santen and Sperling 1985), one would expect the bar conditions would produce stronger effects than the lines conditions, but this is not the case. Moreover, as a result of the aperture problem, every model of motion perception that posits a relationship between spatial orientation and motion direction suggests that an orientationally tuned filter contributes only to motion percepts orthogonal to the filter's preferred spatial orientation. Thus, these models would predict that lines drawn parallel to the indicated path of motion would not influence the motion percept at all because, if there were any effect, the implied motion of these lines, should be orthogonal to the possible motions of the dots. Our results indicate that the lines imply motion parallel to their orientation, so these models of motion perception are lacking. We will return to this issue in experiment 4 below.

\section{Experiment 2: Spatiotemporal relationship}

The results from experiment 1 demonstrate that lines bias an ambiguous motion display in a way consistent with the theory. To further explore this effect we moved the lines and bars from the intervening frames to the same frames as the dots, as schematized in figure 5. In the model, the oriented rebound trail should coexist with the contours generated by the moving stimulus (figure 3 ). Thus, we thought the new display might produce a percept of rotational motion more often than in experiment 1 . Two new naive subjects participated in the experiment.

Figure $5 \mathrm{c}$ shows the average percentage of time across trials that observers reported rotational-motion percepts for the different conditions. The pattern of results is the same as for experiment 1 , but reduced in magnitude. A Tukey test finds that the lines conditions are significantly different from the control condition $(p<0.05)$ but the bar conditions are not.

Clearly, the hypothesis that coexistence of lines and dots would lead to more frequent percepts of rotational motion was not supported. One cannot outright reject this aspect of the model though, because phenomenologically the motion percepts generated by the stimuli in experiment 2 were different from those in experiment 1 . In experiment 1 the dots seemed to move while the bars and lines generally did not and were perceived 
(a)
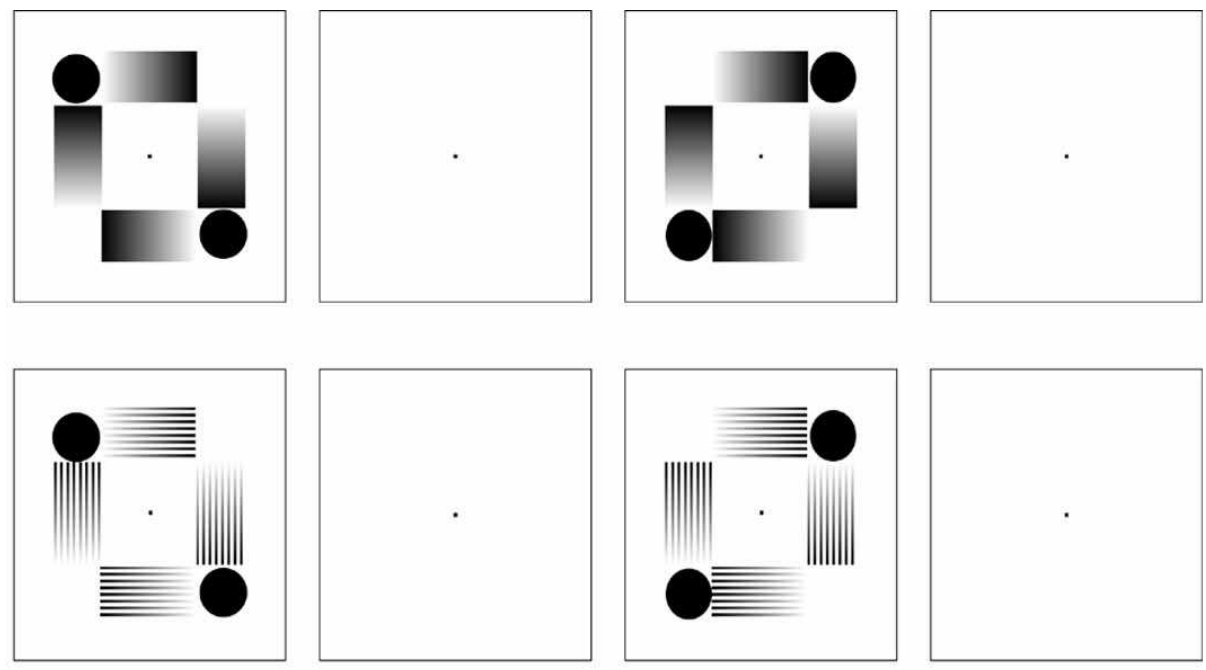

(b)
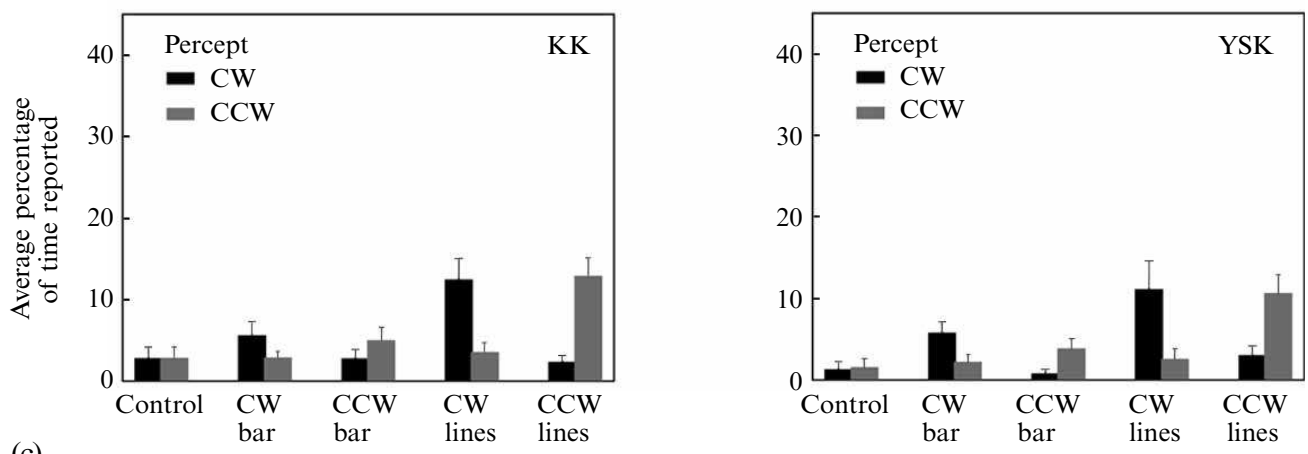

Figure 5. Experiment 2. All stimuli in (a) and (b) are the reverse contrast of what was on the screen. (a) The clockwise (CW) bar stimulus included bars with a clockwise gradient of intensities within each bar. (b) The CW lines stimulus included lines with the same gradient. (c) The percentage of time observers KK and YSK reported seeing motions as indicated for the different stimuli. The control stimulus rarely produced rotational-motion percepts. Though weaker than in experiment 1 , observers saw clockwise rotation for the $\mathrm{CW}$ lines stimuli and counterclockwise $(\mathrm{CCW})$ rotation for the CCW lines stimuli, as predicted. The bar stimuli produced a small, but statistically insignificant, effect. Error bars indicate one standard error of the mean.

as separate from the dots. In experiment 2 the dots and bars/lines often seemed to move together, with the lines and bars seeming to be stretched and pulled by the dots as the entire ensemble moved. Thus, even if there was an effect of coexistence of lines with stimuli, it may have been washed out by the grouping effect of the entire ensemble.

Despite this somewhat disappointing confound, it is worth noting that the pattern of results is the same across the two experiments, thereby indicating the general robustness of the influence of lines.

\section{Experiment 3: Illusory contours}

The rebound trail theory put forth by Kim and Francis (1998) derives from Grossberg and Mingolla's (1985) neural-network model of visual perception. A notable characteristic of that model is that it accounts for the perception of various illusory contours by hypothesizing that excitatory feedback completes a contour between appropriately aligned inducing stimuli. Within this model, the oriented responses making up the 
rebound trail hypothesized by Kim and Francis have a status corresponding roughly to illusory contours in Grossberg and Mingolla's model. Thus, we hypothesized that illusory contours should also be able to influence the ambiguous motion display.

Figure 6 schematizes the stimuli used in this experiment. Illusory contours were introduced with offset line gratings. To construct the illusory contours along the top of figure $6 \mathrm{a}, 13$ equally spaced vertical lines were generated with a thickness of $4.6 \mathrm{~min}$ of arc and a length of $2.08 \mathrm{deg}$. Then every line was partitioned into 6 smaller vertical lines and every other row of these smaller vertical lines was shifted in opposite directions so that adjacent lines were misaligned with respect to each other as in figures $6 \mathrm{a}$ and $6 \mathrm{~b}$. The same approach was used to create the illusory contours in other directions. The luminance of the line inducers was gradually modified to produce the same type of intensity gradients as in experiments 1 and 2 . We hypothesized that the gradient of inducer intensity would produce a gradient of illusory-contour strength. Thus, each side of the frames with illusory contours in figures $6 \mathrm{a}$ and $6 \mathrm{~b}$ should have illusory-contour gradients that increase in a clockwise direction. Such a gradient should imply clockwise rotation. The same naive observers as in experiment 1 were used in experiment 3.

(a)
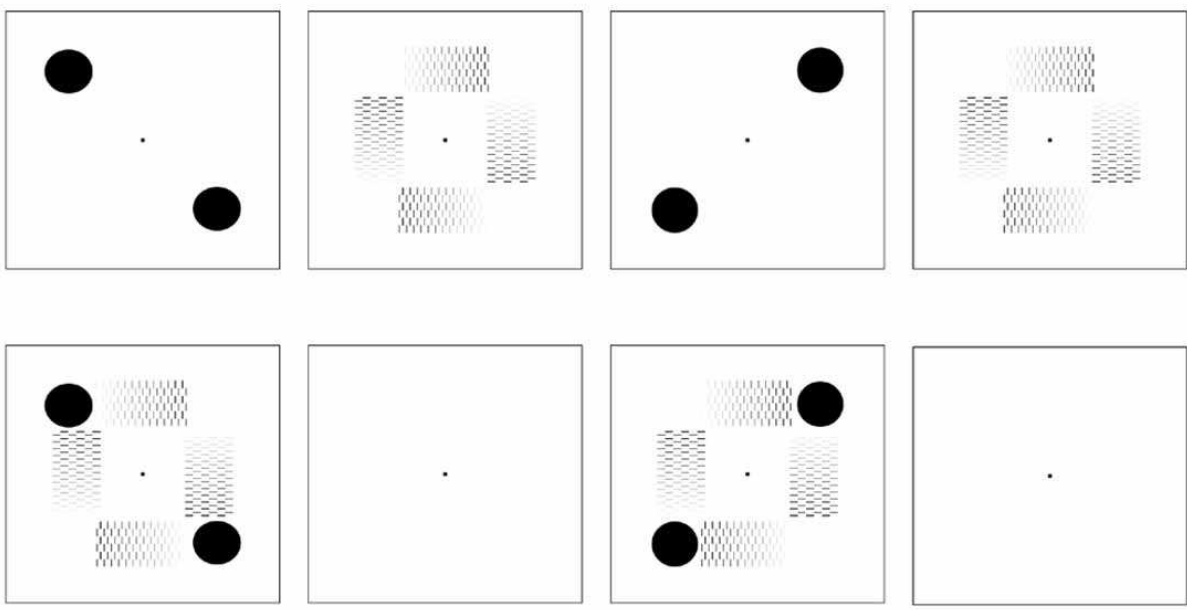

(b)
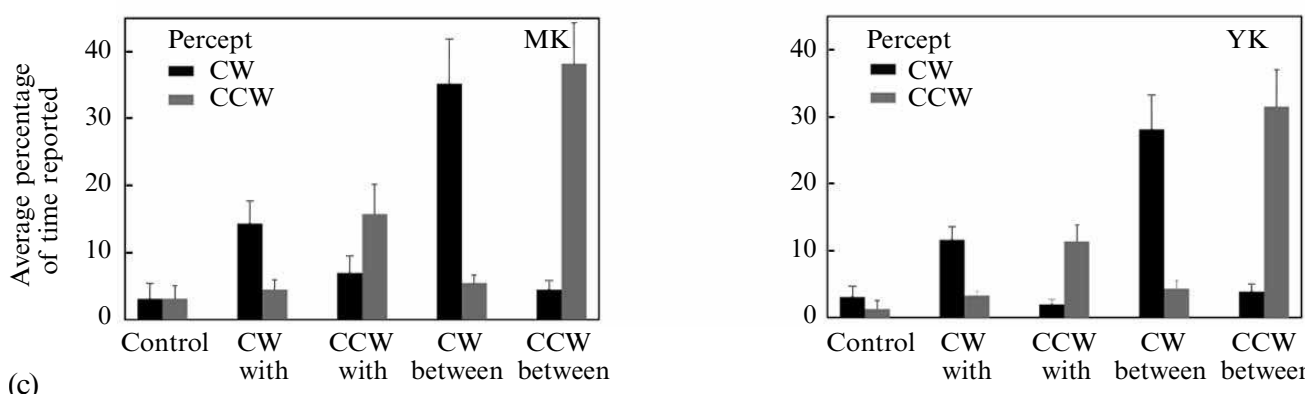

Figure 6. Experiment 3. All stimuli in (a) and (b) are the reverse contrast of what was on the screen. (a) The clockwise $(\mathrm{CW})$ between illusory stimulus included inducers with a clockwise gradient of intensities. The inducers were on the frames between the presentation of the dots. (b) The $\mathrm{CW}$ with stimulus had the same inducers on the frames with the dots. (c) The percentage of time observers MK and YK reported seeing motions as indicated for the different stimuli. The control stimulus rarely produced rotational-motion percepts. Observers saw clockwise rotation for the clockwise lines stimuli and counterclockwise $(\mathrm{CCW})$ rotation for the $\mathrm{CCW}$ stimuli, as predicted. The data for the experimental conditions were significantly different from the control condition. Error bars indicate one standard error of the mean. 
Figure $6 \mathrm{c}$ shows the average percentage of time across trials that observers reported rotational motion. Illusory contours were effective at generating rotational-motion percepts, and the direction of rotation was along the hypothesized gradient of illusorycontour increase. The experimental averages were statistically different from the control condition, both when the line inducers were presented in the frames between the dots and when they were presented in the frames with the dots, although the former produced a much stronger effect. The effect of illusory contours was equivalent to that of the real lines in experiments 1 and 2.

\section{Experiment 4: Orientation preference}

As noted in the discussion of experiment 1, the aperture problem suggests that an oriented filter can reliably indicate only the motion direction orthogonal to its preferred orientation. This property has been incorporated into a variety of models for local motion perception (Adelson and Movshon 1982; Watson and Ahumada 1985; Grossberg and Rudd 1989; Simoncelli and Heeger 1998), with there often being a second stage to calculate a more global motion signal. Indeed, it is well known that orientationally tuned cells in areas V1 and V2 that respond to motion and orientation have an orthogonal relationship between orientation and motion-direction preference (Albright 1984). In contrast, the idea that orientational rebound trails contribute to motion percepts suggests that, at some level of the visual system, cells acting as orientationally tuned filters should also respond to motion directions parallel to their preferred orientation.

The previous experiments suggest that the rebound-trail cue can influence otherwise ambiguous-motion percepts. In this last set of experiments we tested whether lines parallel or orthogonal to the path of motion are more effective at influencing the motion percept. Figures $7 \mathrm{a}$ and $7 \mathrm{~b}$ schematize the type of stimuli we used. Figure $7 \mathrm{a}$ shows stimuli with illusory contours. The inducing elements were identical to those used in experiment 3, except the intensity of all inducers was set to the maximum. In frame 2 of figure $7 \mathrm{a}$, the resulting illusory contours imply horizontal motion, according to the rebound-trail hypothesis. In frame 4 the inducing elements of the top and bottom paths are shifted (every other row moved left or right, respectively) to form complete vertical lines, while the inducing elements of the left and right paths are shifted vertically (every other row moved up or down, respectively) to form vertical illusory contours, thereby implying vertical motion, according to the rebound-trail hypothesis. The effect of these shifts is that the movements of inducers between frames 2 and 4 are ambiguous within a path and equivalent along every path, and thus globally ambiguous as well. However, in the model, the presence of the illusory contours should imply clockwise motion. In contrast, any model that suggests that orientation preference and motiondirection preference are orthogonal would predict that this set of frames should produce counterclockwise motion (if it had any effect) as the dots would be biased to move along the path orthogonal to the orientation of the lines.

In a second experimental condition the illusory contours were replaced with real lines as in figure $7 \mathrm{~b}$. The expectation is the same as for the illusory-contour condition, with this display predicting clockwise motion while models that suppose an orthogonal relationship between preferred spatial orientation and motion direction predicting counterclockwise motion.

Figure $7 \mathrm{c}$ shows the average percentage of time across trials that observers reported rotational motion. For lack of a better approach, the stimuli are defined as clockwise (CW) or counterclockwise (CCW) relative to the prediction of motion based on the rebound-trail hypothesis. The results clearly favor the rebound-trail hypothesis over the prediction of alternative motion theories. That is, the perceived direction of the dots is more often along the path that is drawn with lines parallel to the direction of the motion. 

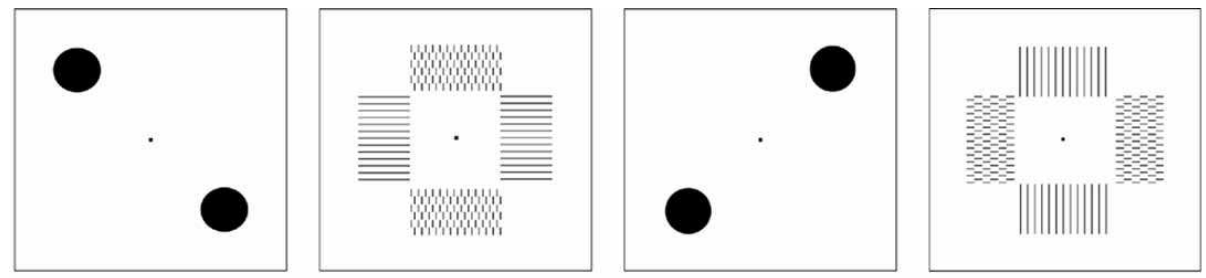

(a)
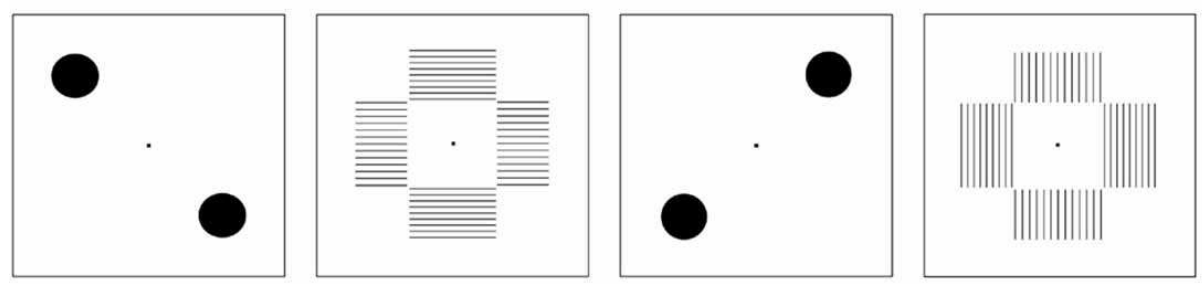

(b)
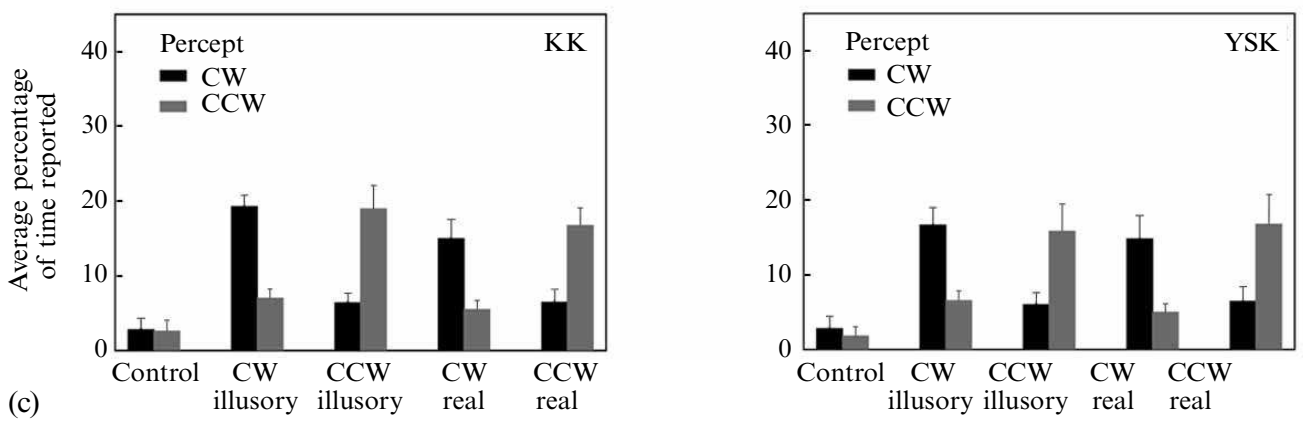

Figure 7. Experiment 4. All stimuli in (a) and (b) are the reverse contrast of what was on the screen. (a) A display (CW illusory) that compares whether motion is seen along a path with illusory contours parallel to motion direction or real contours orthogonal to motion direction. (b) A display ( $\mathrm{CW}$ real) that compares whether motion is seen along a path of real contours parallel to motion direction or a path of real contours orthogonal to motion direction. (c) The percentage of time observers KK and YSK reported seeing motions as indicated for the different stimuli. The control stimulus rarely produced rotational-motion percepts. Observers saw clockwise rotation for the $\mathrm{CW}$ stimuli and counterclockwise $(\mathrm{CCW})$ rotation for the $\mathrm{CCW}$ stimuli, as predicted. The data for the experimental conditions were significantly different from the control condition. Error bars indicate one standard error of the mean.

\section{Conclusions}

The experimental results are consistent with the theory that the visual system is sensitive to oriented rebound trails as a cue to motion. As predicted by the theory, varying the luminance within a line biases motion percepts in the direction of the gradient increase, even though such a gradient in a single bar has very little effect. Illusory contours can also influence the motion percept, and given a choice of moving along a path defined by lines parallel or orthogonal to the path, the perceived movement is more often along the parallel lines.

We know of no other theory of motion perception that can account for these percepts. The significance of the experimental results can be noted by considering other visual phenomena that may be related to the stimulus, but cannot explain the percepts. For example, in a line motion display a dot is briefly flashed and then followed by a line presented to one side of the dot; and the line seems to be drawn from the location of the dot to the line end (Hikosaka et al 1993). In pilot work we did not notice strong effects of line motion, but even if it existed it could not account for the experimental results. 
To see this, consider the effect of line motion on the displays in experiment 1 . If line motion does contribute to the percepts of these displays, then the lines in figure $4 \mathrm{~b}$ that were presented during frames 2 and 4 would generate motion percepts away from the most recently flashed dots. For the lines in frame 2, this would lead to both horizontal clockwise motion and vertical counterclockwise motion. In frame 4 , line motion effects would again lead to both horizontal counterclockwise motion and vertical clockwise motion. Thus, line motion effects, if they had any effect on the percept, are ambiguous with regard to rotational percepts and cannot account for the experimental findings. A modification of line motion theories might suggest that the intensity gradient would produce an internal line motion effect with a motion percept from the more intense side of the line toward the less intense side, but such an effect in our displays would lead to overall percepts in the direction opposite to what we observed experimentally.

Experiments 1 and 2 use stimuli that are somewhat similar to the path-guided-motion experiments of Shepard and Zare (1983). In a two-dot apparent-motion display, they presented a faint gray path connecting the dots during part of the interstimulus interval. Observers reported that they could see the dots move along the path, even when the path curved substantially from a straight line between the dots. Shepard and Zare hypothesized that the gray path was interpreted as blur generated by the moving dot, and thereby contributed to the perceived path of the movement. It seems plausible to us that pathguided-motion and the motion line effects that we found are related. However, we get the strongest effects for lines rather than bars, even though the latter would seem a better representation of blur. Moreover, many of the effects of the path in Shepard and Zare's study can be accounted for by motion-energy types of models, which is not the case for our results. If there is a link between path-guided-motion and our results, we suspect it is that the contours of Shepard and Zare's path act as a motion line trail.

Our experimental results complement a study by Werkhoven et al (1990) on the interaction of orientation and path of motion. Using a different type of ambiguous apparentmotion display with oriented bar elements as the moving stimuli, these authors found that the elements had a strong tendency to move along a path that was parallel to their spatial orientation. The results are in agreement with our finding in experiment 4 that perceived motion tends to be along the lines parallel to the path of motion. They, too, noted that their result seemed inconsistent with the predicted effect of the aperture problem as it would arise in a variety of motion-detection models.

Neural mechanisms sensitive to these orientational cues as indicators of motion may fit into theories of motion processing in area MT (Albright 1984; Movshon et al 1986; Rodman and Albright 1989). Albright (1984) classified motion-sensitive cells in MT as type I or II. Both types of cells showed strong orientation selectivity. Type I cells also showed motion selectivity orthogonal to the preferred orientation. Type II cells showed motion selectivity parallel to the preferred orientation. The properties of type II cells are consistent with the notion that an oriented rebound trail acts as a cue to motion. A type II cell sensitive to this cue will be driven either by a stationary bar parallel to the cell's preferred motion direction, or by movement of an orthogonal bar or spot moving in the preferred direction and thereby leaving a trail of oriented rebounds that are of the cell's preferred orientation. Thus, the lines added in the current experiments might be exciting type II cells, which then influence the systems involved in calculating apparent motion of the dots so as to produce a bias to report motion parallel to the lines.

Additional research (Rodman and Albright 1989) revealed that type II cells were essentially the same as the pattern-motion cells noted by Movshon et al (1986). These pattern-motion cells seemed to respond to the overall pattern of a plaid grating, rather than to its individual components. Two types of computational models have been offered to account for the properties of pattern-motion cells: one is based largely on intersection-of-constraint (IOC) calculations (Albright 1984; Movshon et al 1986; 
Simoncelli and Heeger 1998) and the other is based on feature-tracking calculations (Albright 1984). As Albright (1984) noted, if an oriented contour appears to be stationary when it is part of a moving pattern, then the contour must be moving in a direction parallel to the contour's orientation. Assuming IOC calculations to produce this interpretation take place among type II cells, this would explain why they have parallel orientation and motion direction preferences. However, there is no a priori reason why an IOC calculation needs to assume that a contour that is stationary is moving at all. An equally valid interpretation for IOC models is that a stationary contour is simply stationary and not moving parallel to its orientation. Thus, while IOC models might account for properties of type II cells, and possibly our experimental results, the connection is not straightforward.

An alternative approach based on feature-tracking types of models was also considered by Albright (1984). A motion-sensitive filter can be created out of subunits by properly arranging spatiotemporal lateral inhibition between adjacent subunits. Specifically, if a motion filter receives inhibition from all adjacent subunits but one, it will give its strongest response to motion coming from the direction of the noninhibiting subunit. As Albright showed in computer simulation, such a motion filter also demonstrates a preference for stationary bars oriented along the same direction as the motion preference because this orientation produces the least inhibition. Of course, the properties of the motion-sensitive filter depend on the properties of the subunits. Albright assumed the subunits were defined by circular receptive fields that passed on the intensity of the stimulus within the receptive field. This approach is essentially a version of the wellknown Reichardt motion detectors (Reichardt 1969; van Santen and Sperling 1985). At least as described by Albright, this approach is unlikely to account for our data because we find that illusory contours work as well as luminance-defined contours at influencing motion percepts. The illusory contours seem to be inherently defined as having an orientation and so their effect cannot be explained by Albright's model, at least in its current form.

In contrast to these alternative approaches, our view is that a set of oriented filters aligned along an axis parallel to their preferred orientation acts as a cue to motion in that axis. A gradient among the filters' responses provides a cue for direction along the axis in the direction of the increasing responses. We should note that this claim does not dispute the restrictions of the aperture problem. A single oriented filter cannot detect motion of a contour parallel to its preferred orientation. However, we suggest that a proper set of oriented filters indicates motion parallel to the preferred orientation of those filters. A neurophysiological prediction of this approach is that the rebounding cells in V1 noted by Ringach et al (1997) should project to the pattern-motion cells in MT. Ringach et al found rebounding cells in layers 2, 3, 4B, 5, and 6. Layer 4B and layer 6 of V1 are known to project to MT (see review by Maunsell and van Essen 1983) so that the connection of rebounding cells to type II cells in MT is plausible. Rebounding signals could also take a different route to type II cells in MT by first projecting to area $\mathrm{V} 2$ and then to MT.

Whether or not either of these neurophysiological pathways is responsible for the experimental results, lines can imply motion parallel to their orientation, and the details of how the lines imply motion are consistent with the oriented-rebound-trail hypothesis. Because the predicted existence of the rebound trail is connected to a theory of dynamic vision (Francis et al 1994), and that theory is connected to aspects of spatial vision, texture segmentation, figure-ground distinctions, and brightness perception (Grossberg and Mingolla 1985; Grossberg and Todorović 1988; Grossberg 1994, 1997), the theory demonstrates intricate relationships between areas of visual perception that have previously been considered quite disparate. We anticipate that further analysis of lines as cues for motion will explain a variety of spatiotemporal motion percepts. 


\section{References}

Adelson E H, Bergen J R, 1985 "Spatio-temporal energy models for the perception of motion" Journal of the Optical Society of America A 2 284-299

Adelson E H, Movshon J A, 1982 "Phenomenal coherence of moving visual patterns" Nature (London) $300523-525$

Albright T D, 1984 "Direction and orientation selectivity of neurons in visual area MT of the macaque" Journal of Neurophysiology 52 1106-1130

Carello C, Rosenblum L, Grosofsky A, 1986 "Static depiction of movement" Perception 15 41-58

Francis G, 1996a "Cortical dynamics of lateral inhibition: Visual persistence and ISI" Perception \& Psychophysics $\mathbf{5 8} 1103-1109$

Francis G, 1996b "Cortical dynamics of visual persistence and temporal integration" Perception \& Psychophysics $\mathbf{5 8} 1203-1212$

Francis G, 1997 "Cortical dynamics of lateral inhibition: Metacontrast masking" Psychological Review $104572-594$

Francis G, 1999 "Spatial frequency and visual persistence: Cortical reset" Spatial Vision 12 31-50

Francis G, Grossberg S, 1996a "Cortical dynamics of form and motion integration: Persistence, apparent motion, and illusory contours" Vision Research $36149-174$

Francis G, Grossberg S, 1996b "Cortical dynamics of boundary segmentation and reset: Persistence, afterimages, and residual traces" Perception $25543-567$

Francis G, Grossberg S, Mingolla E, 1994 "Cortical dynamics of feature binding and reset: Control of visual persistence" Vision Research 34 1089-1104

Grossberg S, 1972 "A neural theory of punishment and avoidance: II. Quantitative theory" Mathematical Biosciences $15253-285$

Grossberg S, 1994 "3-D vision and figure-ground separation by visual cortex" Perception \& Psychophysics $\mathbf{5 5} 48-120$

Grossberg S, 1997 "Cortical dynamics of three-dimensional figure-ground perception of twodimension pictures" Psychological Review $104618-658$

Grossberg S, Mingolla E, 1985 "Neural dynamics of perceptual grouping: Textures, boundaries, and emergent segmentations" Perception \& Psychophysics 38 141-171

Grossberg S, Rudd M E, 1989 "A neural architecture for visual motion perception: Group and element apparent motion" Neural Networks $2421-450$

Grossberg S, Todorović D, 1988 "Neural dynamics of 1-D and 2-D brightness perception: A unified model of classical and recent phenomena" Perception \& Psychophysics $43241-277$

Hikosaka O, Miyauchi S, Shimojo S, 1993 "Focal visual-attention produces illusory temporalorder and motion sensation" Vision Research $331219-1240$

Kim H, Francis G, 1998 "A computational and perceptual account of motion lines" Perception $27785-797$

Maunsell J H R, Essen D C van, 1983 "The connections of the middle temporal visual area (MT) and their relationship to a cortical hierarchy in the macaque monkey" Journal of Neuroscience $32563-2586$

Movshon J A, Adelson E H, Gizzi M S, Newsome W T, 1986 "The analysis of moving visual pattern", in Pattern Recognition Mechanisms Eds C Chagas, R Gattass, C Gross (New York: Springer) pp $117-151$

Ramachandran V S, Anstis S M, 1985 "Perceptual organization in multistable apparent motion" Perception $14135-143$

Reichardt W, 1969 "Movement perception in insects", in Processing of Optical Data by Organisms and Machines Ed.W Reichardt (New York: Academic Press) pp 465-493

Ringach D, Hawken M J, Shapley R, 1997 "Dynamics of orientation tuning in macaque primary visual cortex" Nature (London) $\mathbf{3 8 7} 281$-283

Rodman H R, Albright T D, 1989 "Single-unit analysis of pattern-motion selective properties in the middle temporal visual area (MT)" Experimental Brain Research 75 53-64

Santen J P H van, Sperling G, 1985 "Elaborated Reichardt detectors" Journal of the Optical Society of America A $2300-321$

Shepard R N, Zare S L, 1983 "Path-guided apparent motion" Science 220632 -634

Simoncelli E, Heeger D, 1998 "A model of neuronal responses in visual area MT"Vision Research $38743-761$

Watson A B, Ahumada A J, 1985 "Model of human visual-motion sensing" Journal of the Optical Society of America A $2322-342$

Werkhoven P, Snippe H P, Koenderink J J, 1990 "Effects of element orientation on apparent motion perception" Perception \& Psychophysics 47 509-525 
